\title{
Nucleon Tomography and Generalized Parton Distribution at Physical Pion Mass from Lattice QCD
}

\author{
Huey-Wen Lin $\oplus^{1,2, *}$ \\ ${ }^{1}$ Department of Physics and Astronomy, Michigan State University, East Lansing, Michigan 48824, USA \\ ${ }^{2}$ Department of Computational Mathematics, Science and Engineering, Michigan State University, \\ East Lansing, Michigan 48824, USA
}

(Received 8 January 2021; accepted 28 September 2021; published 29 October 2021)

\begin{abstract}
We present the first lattice calculation of the nucleon isovector unpolarized generalized parton distribution at the physical pion mass using a lattice ensemble with $2+1+1$ flavors of highly improved staggered quarks generated by the MILC Collaboration, with lattice spacing $a \approx 0.09 \mathrm{fm}$ and volume $64^{3} \times 96$. We use momentum-smeared sources to improve the signal at nucleon boost momentum $P_{z} \approx$ $2.2 \mathrm{GeV}$ and report results at nonzero momentum transfers in $[0.2,1.0] \mathrm{GeV}^{2}$. Nonperturbative renormalization in a regularization-independent momentum-subtraction scheme is used to obtain the quasidistribution before matching to the light-cone generalized parton distributions. The three-dimensional distributions $H\left(x, Q^{2}\right)$ and $E\left(x, Q^{2}\right)$ at $\xi=0$ are presented, along with the three-dimensional nucleon tomography and impact parameter-dependent distribution for selected Bjorken $x$ at $\mu=3 \mathrm{GeV}$ in a modified minimal subtraction scheme.
\end{abstract}

DOI: $10.1103 /$ PhysRevLett.127.182001

Nucleons (that is, protons and neutrons) are the building blocks of all ordinary matter, and the study of nucleon structure is a central goal of many worldwide experimental efforts. Gluons and quarks are the underlying degrees of freedom that explain the properties of nucleons, and fully understanding how they contribute to the properties of nucleons (such as their mass or spin structure) helps to decode the standard model. In quantum chromodynamics (QCD), gluons strongly interact with themselves and with quarks, binding both nucleons and nuclei. However, due to their confinement within these bound states, we cannot single out individual constituents to study them. More than half a century since the discovery of nucleon structure, our understanding has improved greatly; however, there is still a long way to go in unveiling the nucleon's detailed structure, which is characterized by functions such as the generalized parton distributions (GPDs) [1-3]. GPDs can be viewed as a hybrid of parton distributions (PDFs), form factors, and distribution amplitudes. They play an important role in providing a three-dimensional spatial picture of the nucleon [4] and in revealing its spin structure [2]. Experimentally, GPDs can be accessed in exclusive processes such as deeply virtual Compton scattering [5] or meson production [6]. Experimental collaborations and facilities worldwide have

Published by the American Physical Society under the terms of the Creative Commons Attribution 4.0 International license. Further distribution of this work must maintain attribution to the author(s) and the published article's title, journal citation, and DOI. Funded by SCOAP . been devoted to searching for these last unknowns of the nucleon, including HERMES at DESY, COMPASS at CERN, GSI in Europe, BELLE and JPAC in Japan, Halls $\mathrm{A}, \mathrm{B}$, and $\mathrm{C}$ at Jefferson Laboratory, and PHENIX and STAR at RHIC at Brookhaven National Laboratory in the U.S. There are also plans for future facilities: a U.S. electron-ion collider [7] at Brookhaven National Laboratory, an electronion collider in China [8], and the Large Hadron-Electron Collider in Europe $[9,10]$.

Although interest in GPDs has grown enormously, we still need fresh theoretical and phenomenological ideas, including reliable model-independent techniques. Most QCD models have issues associated with three-dimensional structure that are not yet fully understood, so a reliable framework for extracting three-dimensional parton distributions and fragmentation functions from experimental observables does not yet exist. Theoretically, there are factorization issues in hadron production from hadronic reactions, and theoretical efforts are striving to answer key questions that lie along the path to a precise mapping of three-dimensional nucleon structure from experiment. It has become common understanding that we need to develop a program in both theory and experiment that will allow an accurate flavor decomposition of the nucleon GPDs, including flavor differences in the quark structure, the gluon structure, and the nucleon sea-quark GPDs. Most current theoretical issues are associated with nonperturbative features of QCD, that is, where the strong coupling is too large for analytic perturbative methods to be valid. Using a nonperturbative theoretical method that starts from the quark and gluon degrees of freedom, lattice 
QCD (LQCD), allows us to compute these properties on supercomputers.

Probing hadron structure with LQCD was for many years limited to the first few moments due to complications arising from the breaking of rotational symmetry by the discretized Euclidean spacetime. The breakthrough for LQCD came in 2013, when a technique was proposed to connect quantities calculable on the lattice to those on the light cone. Largemomentum effective theory (LaMET), also known as the "quasi-PDF method" [11-13], allows us to calculate the full Bjorken- $x$ dependence of distributions for the first time. Much progress has been made since the first LaMET paper [13-101]. Most work has been done using only one lattice ensemble, but there has been some progress in determining the size of lattice systematic uncertainties. For example, finite-volume systematics were studied in Refs. [30,83]. Machine-learning algorithms have been applied to the inverse problem [81,102] and to making predictions for larger boost momentum and larger Wilson displacement
[103]. On the lattice discretization errors, a $N_{f}=2+1+1$ superfine $(a \approx 0.042 \mathrm{fm})$ lattice at $310-\mathrm{MeV}$ pion mass was used to study nucleon PDFs in Ref. [88], and results from using multiple lattice spacings were reported in Refs. [86,89,102]. The first attempt to obtain strange and charm distributions of the nucleon was recently reported [87]. However, beyond one-dimensional hadron structure, there is little work available. Last spring, the first lattice study of GPDs was made for pions [31]. During the completion of this work, the ETM Collaboration reported their findings on both unpolarized and polarized nucleon GPDs with largest boost momentum $1.67 \mathrm{GeV}$ at pion mass $M_{\pi} \approx 260 \mathrm{MeV}$ [104]. In this Letter, we present the first LQCD calculation of the nucleon GPD at the physical pion mass using the LaMET method and study the threedimensional structure of the unpolarized nucleon GPDs.

The unpolarized GPDs $H(x, \xi, t)$ and $E(x, \xi, t)$ are defined in terms of the matrix elements

$$
\begin{aligned}
F(x, \xi, t) & =\int \frac{d z^{-}}{4 \pi} e^{i x p^{+} z^{-}}\left\langle p^{\prime \prime}\left|\bar{\psi}\left(-\frac{z}{2}\right) \gamma^{+} L\left(-\frac{z}{2}, \frac{z}{2}\right) \psi\left(\frac{z}{2}\right)\right| p^{\prime}\right\rangle_{z^{+}=0, \vec{z}_{\perp}=0} \\
& =\frac{1}{2 p^{+}}\left[H(x, \xi, t) \bar{u}\left(p^{\prime \prime}\right) \gamma^{+} u\left(p^{\prime}\right)+E(x, \xi, t) \bar{u}\left(p^{\prime \prime}\right) \frac{i \sigma^{+\nu} \Delta_{\nu}}{2 m} u\left(p^{\prime}\right)\right]
\end{aligned}
$$

where $L(-z / 2, z / 2)$ is the gauge link along the light cone and

$\Delta^{\mu}=p^{\prime \mu}-p^{\prime \mu}, \quad t=\Delta^{2}, \quad \xi=\frac{p^{\prime \prime+}-p^{\prime+}}{p^{\prime \prime+}+p^{\prime+}}$.

In the limit $\xi, t \rightarrow 0, H$ reduces to the usual unpolarized parton distributions while the information encoded in $E$ cannot be accessed since they are multiplied by the fourmomentum transfer $\Delta$. Only in exclusive processes with a nonzero momentum transfer can $E$ be probed. The one-loop matching [34,105] for the GPD $H$ and $E$ turns out to be similar to that for the parton distribution.

In this Letter, we focus on the nucleon isovector unpolarized GPDs and their quasi-GPD counterparts defined in terms of spacelike correlations calculated in Breit frame. We use clover valence fermions on an ensemble with lattice spacing $a \approx 0.09 \mathrm{fm}$, spatial (temporal) extent around 5.8 (8.6) fm and with the physical pion mass $M_{\pi} \approx 135 \mathrm{MeV}$ and $N_{f}=2+1+1$ (degenerate up and down, strange, and charm) flavors of highly improved staggered dynamical quarks [106] generated by the MILC Collaboration [107]. The gauge links are one-step hypercubic smeared [108] to suppress discretization effects. The clover parameters are tuned to recover the lowest sea pion mass of the highly improved staggered dynamical quarks. The "mixed-action" approach is commonly used, and there has been promising agreement between the calculated lattice nucleon charges, moments, and form factors and the experimental data when applicable [109-121]. Gaussian momentum smearing [122] is used on the quark field to improve the overlap with ground-state nucleons of the desired boost momentum, allowing us to reach higher boost momenta for the nucleon states. We calculate the matrix elements of the form $\left\langle P_{f}\right| \bar{\psi}[-(z / 2)] \Gamma L[-(z / 2)$, $(z / 2)] \psi(z / 2)\left|P_{i}\right\rangle$ with projection operators $\left[\left(1+\gamma_{t}\right) / 2\right] \times$ $\left(1+i \gamma_{5} \gamma_{x, y, z}\right)$. We also use high-statistics measurements, 501760 total over 1960 configurations, to drive down the increased statistical noise at high boost momenta, $P_{z}=$ $\left|\left(\vec{P}_{i}+\vec{P}_{f}\right) / 2\right|=\left|(2 \pi / L)\{0,0,10\} a^{-1}\right|$, and vary spatial momentum transfer $\vec{q}=\vec{P}_{f}-\vec{P}_{i}=(2 \pi / L)\left\{n_{x}, n_{y}, 0\right\} a^{-1}$ with integer $n_{x, y}$ and $n_{x}^{2}+n_{y}^{2} \in\{0,4,8,16,20\}$ with fourmomentum transfer squared $Q^{2}=-q_{\mu} q^{\mu}=\{0,0.19$, $0.39,0.77,0.97\} \mathrm{GeV}^{2}$. We solve a set of linear equations to obtain $H$ and $E$ (similar to form-factor extraction) with all $|q|$ at fixed $Q^{2}$ and rotationally averaged. Technical details (such as renormalization) and more information on how the matrix elements are extracted can be found in the Supplemental Material [123] and our previous work $[23,26,28,126]$.

The nonperturbatively renormalized matrix elements are then Fourier transformed into quasi-GPDs through two different approaches. Following the recent work $[23,26,28]$, we take the matrix elements $z \in[-12,12]$ and apply the simple but effective "derivative" method, 
$\tilde{Q}=i \int_{-z_{\max }}^{+z_{\max }} d z e^{i x P_{z} z} \tilde{h}_{R}^{\prime} / x$, to obtain the quasi-GPDs. Alternatively, we adopt the extrapolation formulation suggested by Ref. [127] by fitting $|z| \in\{10,15\}$ using the formula $c_{1}\left(-i z P_{z}\right)^{-d_{1}}+c_{2} e^{i z P_{z}}\left(i z P_{z}\right)^{-d_{2}}$, inspired by the Regge behavior, to extrapolate the matrix elements into the region beyond the lattice calculation and suppress Fourier-transformation artifacts. Then, both quasi-GPDs are matched to the physical GPDs by applying the matching condition $[23,26,39,65]$. Examples of the GPDs at momentum transfer $Q^{2} \approx 0.4 \mathrm{GeV}^{2}$ are shown in Fig. 1 . Figure 1 compares the $H$ and $E$ GPDs at $Q^{2} \approx 0.4 \mathrm{GeV}^{2}$ with the quasidistribution and matched distribution using $P_{z} \approx 2.2 \mathrm{GeV}$. The matching lowers the positive mid- $x$ to large- $x$ distribution, as expected; as one approaches the light-cone limit, the probability of a parton carrying a larger fraction of its parent nucleon's momentum should become smaller. We also find that the derivative and Regge-inspired extrapolation agree in the mid- to large- $x$ regions, but their difference grows as $x$ approaches zero in both the quark and antiquark distribution. This is expected, since they differ mainly in the treatment of the large- $z$ matrix elements in the quasi-GPD Fourier transformation, which contributes more
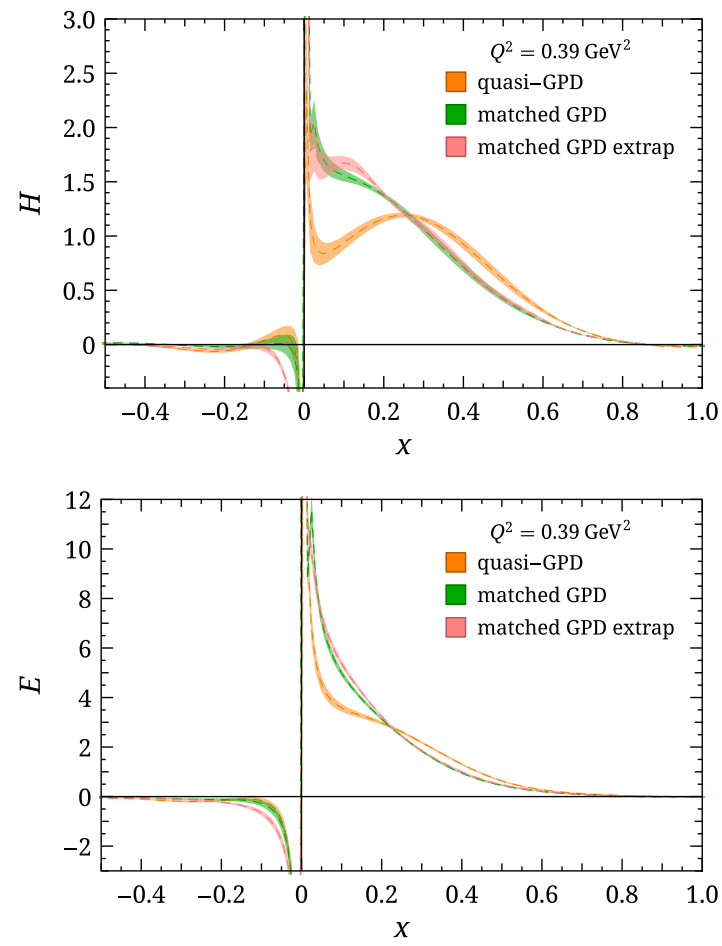

FIG. 1. Nucleon isovector $H$ and $E$ quasi-GPDs and matched GPDs at momentum transfer $Q^{2}=0.39 \mathrm{GeV}^{2}$. The orange and green bands are the quasi-GPD and matched GPDs from the derivative method [17], while the pink band corresponds to the matched GPD using quasi-GPD from the extrapolation formulation suggested by Ref. [127]. We find both methods give reasonable agreement in the $x$-dependent behavior, except in the small- $x$ region, which is dominated by the large- $z$ matrix elements that rely on the extrapolation. significantly to the small- $x$ distribution. By repeating a similar analysis for each available $Q^{2}$ in this calculation, we can construct the full three-dimensional shape of $H$ and $E$ as functions of $x$ and $Q^{2}$, as shown in Fig. 2. Because of the limited reliable $z P_{z}$ reach of the lattice calculation, we find that the small- $x$ region is unreliable due to lack of precision lattice data to constrain it. Thus, due to charge conservation, the antiquark (negative- $x$ ) distribution can also be sensitive to $P_{z}$. It has been found in past work $[17,23,26,28]$ that higher boost momenta are needed to improve the antiquark region. Therefore, for the rest of this Letter, we will mainly focus on the $x>0.05$ region. For convenience, we will focus on showing the GPD results from the derivative method and use the Regge-inspired extrapolation to estimate the uncertainties in the small- $x$ region by reconstructing GPD moments from our $x$-dependent GPD functions.

Since this is the first lattice calculation with full threedimensional $x$ and $Q^{2}$ dependence of the $H$ and $E$ GPD functions, we would like to check how the new results using the LaMET approach compare with the previous moment approaches to the generalized form factors. In the $\xi \rightarrow 0$ limit, the $H$ and $E$ GPDs decrease monotonically as $x$ or $Q^{2}$ increases. We take Mellin moments of the GPDs to compare with previous lattice calculations done using local matrix elements through the operator product expansion (OPE). Taking the $x$ moments of $H$ and $E[128,129]$,
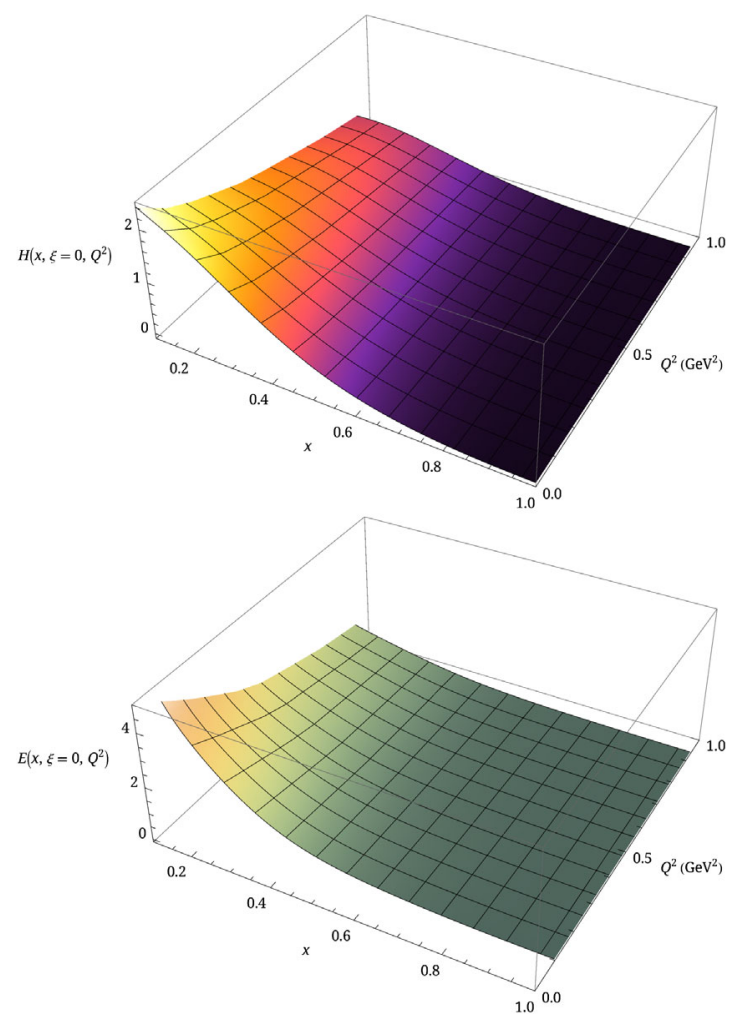

FIG. 2. Nucleon isovector $H$ and $E$ GPDs at $\xi=0$ as functions of $x$ and momentum transfer $Q^{2}$. 


$$
\begin{aligned}
& \int_{-1}^{+1} d x x^{n-1} H\left(x, \xi, Q^{2}\right) \\
& =\sum_{i=0, \text { even }}^{n-1}(-2 \xi)^{i} A_{n i}\left(Q^{2}\right)+\left.(-2 \xi)^{n} C_{n 0}\left(Q^{2}\right)\right|_{n \text { even }}, \\
& \int_{-1}^{+1} d x x^{n-1} E\left(x, \xi, Q^{2}\right) \\
& =\sum_{i=0, \text { even }}^{n-1}(-2 \xi)^{i} B_{n i}\left(Q^{2}\right)-\left.(-2 \xi)^{n} C_{n 0}\left(Q^{2}\right)\right|_{n \text { even }},
\end{aligned}
$$

where the generalized form factors (GFFs) $A_{n i}\left(Q^{2}\right)$, $B_{n i}\left(Q^{2}\right)$, and $C_{n i}\left(Q^{2}\right)$ in the $\xi$ expansion on the right-hand side are real functions. When $n=1$, we get the Dirac and Pauli electromagnetic form factors $F_{1}\left(Q^{2}\right)=A_{10}\left(Q^{2}\right)$ and $F_{2}\left(Q^{2}\right)=B_{10}\left(Q^{2}\right)$. To compare with other lattice results, we plot the Sachs electric and magnetic form factors using $F_{1,2}$ as $G_{E}\left(Q^{2}\right)=F_{1}\left(Q^{2}\right)+q^{2} F_{2}\left(Q^{2}\right) /\left(2 M_{N}\right)^{2}$ and $G_{M}\left(Q^{2}\right)=F_{1}\left(Q^{2}\right)+F_{2}\left(Q^{2}\right)$ in Fig. 3 with selected results from near-physical pion masses. The PACS has the largest volume among these calculations and is able to probe the smallest $Q^{2}$. Overall, our results are not only consistent within errors with the earlier PNDME Collaboration study using the same ensemble (but which used local operators) but are also in good agreement with other lattice collaborations. When $n=2$, we obtained GFFs of $A_{20}\left(Q^{2}\right)$ and $B_{20}\left(Q^{2}\right)$ so that we can compare
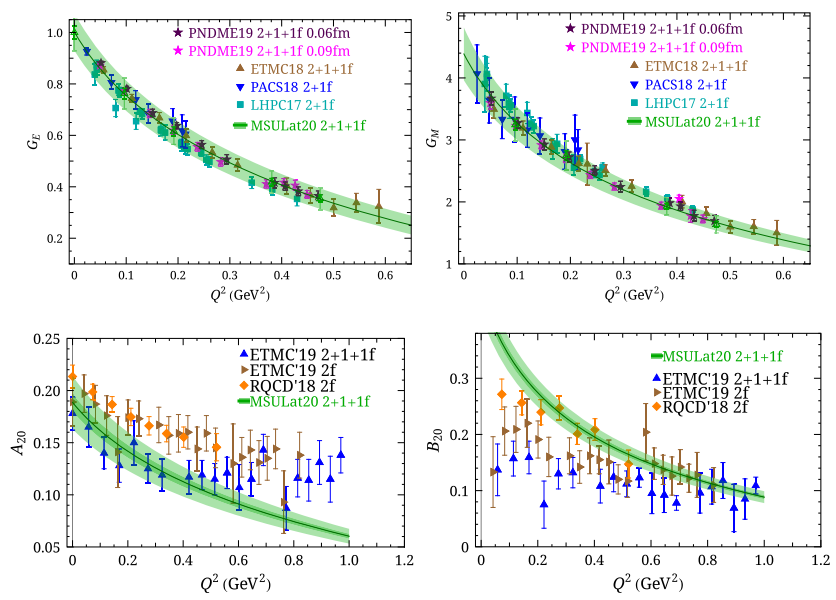

FIG. 3. (Top) The nucleon isovector electric and magnetic form factor results obtained from this work (labeled as "MSULat20 $2+1+1$ ”) as functions of transferred momentum $Q^{2}$, and comparison with other lattice works calculated near physical pion mass: $N_{f}=2$ ETMC18 [132] $N_{f}=2+1$ LHPC14 [133], LHPC17 [134], PACS18 [135]; $N_{f}=2+1+1$ ETMC18 [136], PNDME19 [137] with 2 lattice spacings of 0.06 and $0.09 \mathrm{fm}$. (Bottom) The unpolarized nucleon isovector GFFs obtained from this work, compared with other lattice results calculated near physical pion mass as functions of transfer momentum $Q^{2}$ : ETMC19 [130], and RQCD19 [131]. our moment results with past lattice calculations using the OPE, as shown in Fig. 3. We compare our moment results with those obtained from simulations at the physical point by the European Twisted Mass Collaboration (ETMC) using three ensembles [130] and the near-physical calculation of the RQCD Collaboration [131]. We note that even with the same OPE approach by the same collaboration, the two datasets for $A_{20}$ in the ETMC calculation exhibit some tension. This is an indication that the systematic uncertainties are more complicated for these GFFs. Given that the blue points correspond to finer lattice spacing, larger volume, and larger $m_{\pi} L$, we expect that the blue points have suppressed systematic uncertainties. Our moment result $A_{20}\left(Q^{2}\right)$ is in better agreement with those obtained using the OPE approach at small momentum transfer $Q^{2}$, while $B_{20}\left(Q^{2}\right)$ is in better agreement with OPE approaches at large $Q^{2}$. The comparison between the $N_{f}=2$ ETMC data and $N_{f}=2$ RQCD data reveals agreement for $A_{20}$ and $B_{20}$. However, the RQCD data have a different slope than the ETMC data, which is attributed to the different analysis methods and systematic uncertainties. Both our results and ETMC's are done using a single ensemble; future studies to include other lattice artifacts, such as lattice-spacing dependence, are important to account for the difference in the results.

Note that the error bands in Fig. 3 include the systematics from the following: (1) The systematics due to the negative$x$ and small- $x$ regions of the current GPD extraction. We create lattice pseudoata using input of CT18NNLO PDF [138] with the same lattice parameters (such as $z$ and $P_{z}$ ) used in this calculation and apply the same analysis procedure described above. We take the upper limit of the reconstructed and original CT18 moments as an estimate of the systematics introduced by the analysis procedure (e.g., by Fourier truncation). (2) We vary the maximum Wilson-line length $z$ within 2 lattice units and take half the difference as an estimate of the systematic due to finite $z$. (3) We estimate $1 / P_{z}$ systematics due to highertwist effects by comparing our $Q^{2}=0$ PDFs and to those in the previous works with 3 boost momenta $[23,26]$. The final errors are summed in quadrature to create the final error bands shown in Fig. 3.

The Fourier transform of the nonspin-flip GPD $H\left(x, \xi=0, Q^{2}\right)$ gives the impact parameter-dependent distribution $\mathrm{q}(x, b)[139]$

$$
\mathrm{q}(x, b)=\int \frac{d \boldsymbol{q}}{(2 \pi)^{2}} H\left(x, \xi=0, t=-\boldsymbol{q}^{2}\right) e^{i \boldsymbol{q} \cdot \mathbf{b}},
$$

where $b$ is the transverse distance from the center of momentum. Figure 4 shows the first results of impact parameter-dependent distribution from LQCD: a threedimensional distribution as function of $x$ and $b$, and two-dimensional distributions at $x=0.3,0.5$, and 0.7 . The impact parameter-dependent distribution describes 

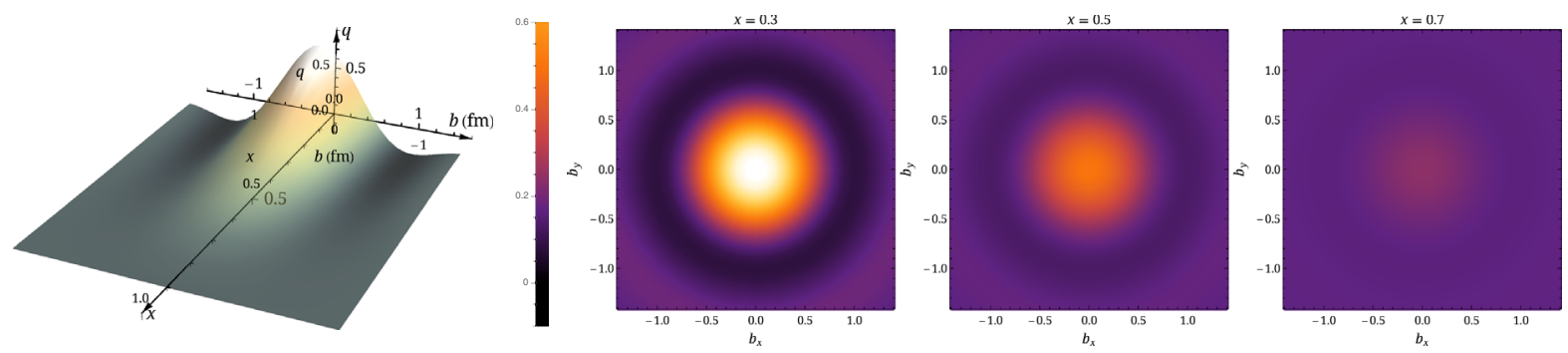

FIG. 4. (Left) Nucleon tomography: three-dimensional impact parameter-dependent parton distribution as a function of $x$ and $b$ using lattice $H$ at physical pion mass. (Right) The two-dimensional impact-parameter-dependent distribution for $x=0.3$, 0.5, and 0.7 .

the probability density for a parton with momentum fraction $x$ at distance $b$ in the transverse plane. Figure 4 shows that the probability decreases quickly as $x$ increases. Using Eq. (4) and the $H\left(x, \xi=0, t=-q^{2}\right)$ obtained from the lattice calculation at the physical pion mass, we can take a snapshot of the nucleon in the transverse plane to perform $x$-dependent nucleon tomography using LQCD for the first time.

In this Letter, we describe our computation of the isovector nucleon unpolarized GPDs at physical pion mass using boost momentum $2.2 \mathrm{GeV}$ with nonzero momentum transfers in $[0.2,1.0] \mathrm{GeV}^{2}$. We show that we are able to map out the first three-dimensional GPD structures using lattice QCD in the special limit $\xi=0$. There are residual lattice systematics that are not yet included in the current calculation. In our past studies, we found the finite-volume effects to be negligible for isovector nucleon quasidistributions calculated within the range $M_{\pi}^{\mathrm{val}} L \in\{3.3,5.5\}$. We anticipate such systematics should be small compared to the statistical errors. The lattice discretization has been studied by the MSULat Collaboration in Refs. [86,102] with multiple lattice spacings in the LaMET study of pion and kaon distribution amplitudes and PDFs; similarly, a comparison of nucleon isovector PDFs with 0.045 and $0.12 \mathrm{fm}$ lattice spacing is shown in the Supplemental Material [123]. There was mild lattice-spacing dependence for a majority of the Wilson-link displacements studied with similar largest boost momenta with same valence or sea lattice setup. ETMC also reports LaMET isovector nucleon PDFs in Ref. [140] using twisted-mass fermion actions and reports different findings. Future work will investigate ensembles with smaller lattice spacing to reach even higher boost momentum (either directly or with the aid of machine learning [103]) so that we can push toward reliable determination of the smaller- $x$ and antiquark regions.

We thank the MILC Collaboration for sharing the lattices used to perform this study. The LQCD calculations were performed using the CHROMA software suite [141]. This research used resources of the National Energy Research Scientific Computing Center, a DOE Office of Science User Facility supported by the Office of Science of the
U.S. Department of Energy under Contract No. DE-AC0205CH11231 through ERCAP; facilities of the USQCD Collaboration, which are funded by the Office of Science of the U.S. Department of Energy, and supported in part by Michigan State University through computational resources provided by the Institute for Cyber-Enabled Research (iCER). The work of H. L. is partially supported by the U.S. National Science Foundation under Grant No. PHY 1653405 "CAREER: Constraining Parton Distribution Functions for New-Physics Searches" and by the Research Corporation for Science Advancement through the Cottrell Scholar Award "Unveiling the ThreeDimensional Structure of Nucleons."

* Corresponding author. hwlin@pa.msu.edu

[1] D. Mller, D. Robaschik, B. Geyer, F.-M. Dittes, and J. Hořejši, Fortschr. Phys. 42, 101 (1994).

[2] X.-D. Ji, Phys. Rev. Lett. 78, 610 (1997).

[3] A. Radyushkin, Phys. Lett. B 380, 417 (1996).

[4] M. Burkardt, Phys. Rev. D 62, 071503(R) (2000); 66, 119903(E) (2002).

[5] X.-D. Ji, Phys. Rev. D 55, 7114 (1997).

[6] B. Kriesten, A. Meyer, S. Liuti, L. C. Diaz, D. Keller, G. R. Goldstein, and J. O. Gonzalez-Hernandez, Phys. Rev. D 101, 054021 (2020).

[7] National Academies of Sciences and Medicine, An Assessment of U.S.-Based Electron-Ion Collider Science (The National Academies Press, Washington, DC, 2018), https://doi.org/10.17226/25171.

[8] D. P. Anderle et al., Front. Phys. (Beijing) 16, 64701 (2021).

[9] J. A. Fernandez et al. (LHeC Study Group), J. Phys. G 39, 075001 (2012).

[10] P. Agostini et al. (LHeC and FCC-he Study Group), arXiv:2007.14491.

[11] X. Ji, Phys. Rev. Lett. 110, 262002 (2013).

[12] X. Ji, Sci. China Phys. Mech. Astron. 57, 1407 (2014).

[13] X. Ji, J.-H. Zhang, and Y. Zhao, Nucl. Phys. B924, 366 (2017).

[14] H.-W. Lin, Proc. Sci., LATTICE2013 (2014) 293.

[15] H.-W. Lin, J.-W. Chen, S. D. Cohen, and X. Ji, Phys. Rev. D 91, 054510 (2015). 
[16] J.-W. Chen, S. D. Cohen, X. Ji, H.-W. Lin, and J.-H. Zhang, Nucl. Phys. B911, 246 (2016).

[17] H.-W. Lin, J.-W. Chen, T. Ishikawa, and J.-H. Zhang (LP3 Collaboration), Phys. Rev. D 98, 054504 (2018).

[18] C. Alexandrou, K. Cichy, V. Drach, E. Garcia-Ramos, K. Hadjiyiannakou, K. Jansen, F. Steffens, and C. Wiese, Phys. Rev. D 92, 014502 (2015).

[19] C. Alexandrou, K. Cichy, M. Constantinou, K. Hadjiyiannakou, K. Jansen, F. Steffens, and C. Wiese, Phys. Rev. D 96, 014513 (2017).

[20] C. Alexandrou, K. Cichy, M. Constantinou, K. Hadjiyiannakou, K. Jansen, H. Panagopoulos, and F. Steffens, Nucl. Phys. B923, 394 (2017).

[21] J.-W. Chen, T. Ishikawa, L. Jin, H.-W. Lin, Y.-B. Yang, J.-H. Zhang, and Y. Zhao, Phys. Rev. D 97, 014505 (2018).

[22] C. Alexandrou, K. Cichy, M. Constantinou, K. Jansen, A. Scapellato, and F. Steffens, Phys. Rev. Lett. 121, 112001 (2018).

[23] J.-W. Chen, L. Jin, H.-W. Lin, Y.-S. Liu, Y.-B. Yang, J.-H. Zhang, and Y. Zhao, arXiv:1803.04393.

[24] J.-H. Zhang, J.-W. Chen, L. Jin, H.-W. Lin, A. Schäfer, and Y. Zhao, Phys. Rev. D 100, 034505 (2019).

[25] C. Alexandrou, K. Cichy, M. Constantinou, K. Jansen, A. Scapellato, and F. Steffens, Phys. Rev. D 98, 091503(R) (2018).

[26] H.-W. Lin, J.-W. Chen, X. Ji, L. Jin, R. Li, Y.-S. Liu, Y.-B. Yang, J.-H. Zhang, and Y. Zhao, Phys. Rev. Lett. 121, 242003 (2018).

[27] Z.-Y. Fan, Y.-B. Yang, A. Anthony, H.-W. Lin, and K.-F. Liu, Phys. Rev. Lett. 121, 242001 (2018).

[28] Y.-S. Liu, J.-W. Chen, L. Jin, R. Li, H.-W. Lin, Y.-B. Yang, J.-H. Zhang, and Y. Zhao, arXiv:1810.05043.

[29] W. Wang, J.-H. Zhang, S. Zhao, and R. Zhu, Phys. Rev. D 100, 074509 (2019).

[30] H.-W. Lin and R. Zhang, Phys. Rev. D 100, 074502 (2019).

[31] J.-W. Chen, H.-W. Lin, and J.-H. Zhang, arXiv: 1904.12376.

[32] X. Xiong, X. Ji, J.-H. Zhang, and Y. Zhao, Phys. Rev. D 90, 014051 (2014).

[33] X. Ji and J.-H. Zhang, Phys. Rev. D 92, 034006 (2015).

[34] X. Ji, A. Schäfer, X. Xiong, and J.-H. Zhang, Phys. Rev. D 92, 014039 (2015).

[35] X. Xiong and J.-H. Zhang, Phys. Rev. D 92, 054037 (2015).

[36] X. Ji, P. Sun, X. Xiong, and F. Yuan, Phys. Rev. D 91, 074009 (2015).

[37] C. Monahan, Phys. Rev. D 97, 054507 (2018).

[38] X. Ji, L.-C. Jin, F. Yuan, J.-H. Zhang, and Y. Zhao, Phys. Rev. D 99, 114006 (2019).

[39] I. W. Stewart and Y. Zhao, Phys. Rev. D 97, 054512 (2018).

[40] M. Constantinou and H. Panagopoulos, Phys. Rev. D 96, 054506 (2017).

[41] J. Green, K. Jansen, and F. Steffens, Phys. Rev. Lett. 121, 022004 (2018).

[42] T. Izubuchi, X. Ji, L. Jin, I. W. Stewart, and Y. Zhao, Phys. Rev. D 98, 056004 (2018).

[43] X. Xiong, T. Luu, and U.-G. Meiner, arXiv:1705.00246.

[44] W. Wang, S. Zhao, and R. Zhu, Eur. Phys. J. C 78, 147 (2018).
[45] W. Wang and S. Zhao, J. High Energy Phys. 05 (2018) 142.

[46] J. Xu, Q.-A. Zhang, and S. Zhao, Phys. Rev. D 97, 114026 (2018).

[47] J.-H. Zhang, J.-W. Chen, X. Ji, L. Jin, and H.-W. Lin, Phys. Rev. D 95, 094514 (2017).

[48] T. Ishikawa, Y.-Q. Ma, J.-W. Qiu, and S. Yoshida, arXiv:1609.02018.

[49] J.-W. Chen, X. Ji, and J.-H. Zhang, Nucl. Phys. B915, 1 (2017).

[50] X. Ji, J.-H. Zhang, and Y. Zhao, Phys. Rev. Lett. 120, 112001 (2018).

[51] T. Ishikawa, Y.-Q. Ma, J.-W. Qiu, and S. Yoshida, Phys. Rev. D 96, 094019 (2017).

[52] T. Ishikawa, L. Jin, H.-W. Lin, A. Schfer, Y.-B. Yang, J.-H. Zhang, and Y. Zhao, Sci. China Phys. Mech. Astron. 62, 991021 (2019).

[53] H.-N. Li, Phys. Rev. D 94, 074036 (2016).

[54] C. Monahan and K. Orginos, J. High Energy Phys. 03 (2017) 116.

[55] A. Radyushkin, Phys. Lett. B 767, 314 (2017).

[56] G. C. Rossi and M. Testa, Phys. Rev. D 96, 014507 (2017).

[57] C. E. Carlson and M. Freid, Phys. Rev. D 95, 094504 (2017).

[58] R. A. Briceño, J. V. Guerrero, M. T. Hansen, and C. J. Monahan, Phys. Rev. D 98, 014511 (2018).

[59] T. J. Hobbs, Phys. Rev. D 97, 054028 (2018).

[60] Y. Jia, S. Liang, L. Li, and X. Xiong, J. High Energy Phys. 11 (2017) 151.

[61] S.-S. Xu, L. Chang, C. D. Roberts, and H.-S. Zong, Phys. Rev. D 97, 094014 (2018).

[62] Y. Jia, S. Liang, X. Xiong, and R. Yu, Phys. Rev. D 98, 054011 (2018).

[63] G. Spanoudes and H. Panagopoulos, Phys. Rev. D 98, 014509 (2018).

[64] G. C. Rossi and M. Testa, Phys. Rev. D 98, 054028 (2018).

[65] Y.-S. Liu, J.-W. Chen, L. Jin, H.-W. Lin, Y.-B. Yang, J.-H. Zhang, and Y. Zhao, Phys. Rev. D 101, 034020 (2020).

[66] X. Ji, Y. Liu, and I. Zahed, Phys. Rev. D 99, 054008 (2019).

[67] S. Bhattacharya, C. Cocuzza, and A. Metz, Phys. Lett. B 788, 453 (2019).

[68] A. V. Radyushkin, Phys. Lett. B 788, 380 (2019).

[69] J.-H. Zhang, X. Ji, A. Schäfer, W. Wang, and S. Zhao, Phys. Rev. Lett. 122, 142001 (2019).

[70] Z.-Y. Li, Y.-Q. Ma, and J.-W. Qiu, Phys. Rev. Lett. 122, 062002 (2019).

[71] V. M. Braun, A. Vladimirov, and J.-H. Zhang, Phys. Rev. D 99, 014013 (2019).

[72] W. Detmold, R. G. Edwards, J. J. Dudek, M. Engelhardt, H.-W. Lin, S. Meinel, K. Orginos, and P. Shanahan (USQCD Collaboration), Eur. Phys. J. A 55, 193 (2019).

[73] M. A. Ebert, I. W. Stewart, and Y. Zhao, J. High Energy Phys. 03 (2020) 099.

[74] X. Ji, Y. Liu, and Y.-S. Liu, Phys. Lett. B 811, 135946 (2020).

[75] G. S. Bali et al., Eur. Phys. J. C 78, 217 (2018).

[76] G. S. Bali, V. M. Braun, B. Gläßle, M. Göckeler, M. Gruber, F. Hutzler, P. Korcyl, A. Schäfer, P. Wein, and J.-H. Zhang, Phys. Rev. D 98, 094507 (2018). 
[77] R. S. Sufian, J. Karpie, C. Egerer, K. Orginos, J.-W. Qiu, and D. G. Richards, Phys. Rev. D 99, 074507 (2019).

[78] G. S. Bali et al. (RQCD Collaboration), Eur. Phys. J. A 55, 116 (2019).

[79] K. Orginos, A. Radyushkin, J. Karpie, and S. Zafeiropoulos, Phys. Rev. D 96, 094503 (2017).

[80] J. Karpie, K. Orginos, and S. Zafeiropoulos, J. High Energy Phys. 11 (2018) 178.

[81] J. Karpie, K. Orginos, A. Rothkopf, and S. Zafeiropoulos, J. High Energy Phys. 04 (2019) 057.

[82] B. Jo, J. Karpie, K. Orginos, A. Radyushkin, D. Richards, and S. Zafeiropoulos, J. High Energy Phys. 12 (2019) 081.

[83] B. Joó, J. Karpie, K. Orginos, A. V. Radyushkin, D. G. Richards, R. S. Sufian, and S. Zafeiropoulos, Phys. Rev. D 100, 114512 (2019).

[84] A. Radyushkin, Phys. Rev. D 98, 014019 (2018).

[85] J.-H. Zhang, J.-W. Chen, and C. Monahan, Phys. Rev. D 97, 074508 (2018).

[86] H.-W. Lin, J.-W. Chen, Z. Fan, J.-H. Zhang, and R. Zhang, Phys. Rev. D 103, 014516 (2021).

[87] R. Zhang, H.-W. Lin, and B. Yoon, arXiv:2005.01124 [Phys. Rev. D (to be published)].

[88] Z. Fan, X. Gao, R. Li, H.-W. Lin, N. Karthik, S. Mukherjee, P. Petreczky, S. Syritsyn, Y.-B. Yang, and R. Zhang, Phys. Rev. D 102, 074504 (2020).

[89] R. S. Sufian, C. Egerer, J. Karpie, R. G. Edwards, B. Jo, Y.-Q. Ma, K. Orginos, J.-W. Qiu, and D. G. Richards, Phys. Rev. D 102, 054508 (2020).

[90] X. Gao, L. Jin, C. Kallidonis, N. Karthik, S. Mukherjee, P. Petreczky, C. Shugert, S. Syritsyn, and Y. Zhao, Phys. Rev. D 102, 094513 (2020).

[91] J. R. Green, K. Jansen, and F. Steffens, Phys. Rev. D 101, 074509 (2020).

[92] Y. Chai et al., Phys. Rev. D 102, 014508 (2020).

[93] P. Shanahan, M. Wagman, and Y. Zhao, Phys. Rev. D 102, 014511 (2020).

[94] V. Braun, K. Chetyrkin, and B. Kniehl, J. High Energy Phys. 07 (2020) 161.

[95] S. Bhattacharya, K. Cichy, M. Constantinou, A. Metz, A. Scapellato, and F. Steffens, Phys. Rev. D 102, 111501 (2020).

[96] X. Ji, Y.-S. Liu, Y. Liu, J.-H. Zhang, and Y. Zhao, Rev. Mod. Phys. 93, 035005 (2021).

[97] M. A. Ebert, S. T. Schindler, I. W. Stewart, and Y. Zhao, J. High Energy Phys. 09 (2020) 099.

[98] H.-W. Lin, Int. J. Mod. Phys. A 35, 2030006 (2020).

[99] B. Jo, J. Karpie, K. Orginos, A. V. Radyushkin, D. G. Richards, and S. Zafeiropoulos, Phys. Rev. Lett. 125, 232003 (2020).

[100] M. Bhat, K. Cichy, M. Constantinou, and A. Scapellato, Phys. Rev. D 103, 034510 (2021).

[101] Z. Fan, R. Zhang, and H.-W. Lin, Int. J. Mod. Phys. A 36, 2150080 (2021).

[102] R. Zhang, C. Honkala, H.-W. Lin, and J.-W. Chen, Phys. Rev. D 102, 094519 (2020).

[103] R. Zhang, Z. Fan, R. Li, H.-W. Lin, and B. Yoon, Phys. Rev. D 101, 034516 (2020).

[104] C. Alexandrou, K. Cichy, M. Constantinou, K. Hadjiyiannakou, K. Jansen, A. Scapellato, and F. Steffens, Phys. Rev. Lett. 125, 262001 (2020).
[105] Y.-S. Liu, W. Wang, J. Xu, Q.-A. Zhang, J.-H. Zhang, S. Zhao, and Y. Zhao, Phys. Rev. D 100, 034006 (2019).

[106] E. Follana, Q. Mason, C. Davies, K. Hornbostel, G. P. Lepage, J. Shigemitsu, H. Trottier, and K. Wong (HPQCD and UKQCD Collaborations), Phys. Rev. D 75, 054502 (2007).

[107] A. Bazavov, C. Bernard, J. Komijani, C. DeTar, L. Levkova, W. Freeman, S. Gottlieb, R. Zhou, U. M. Heller, J. E. Hetrick, J. Laiho, J. Osborn, R. L. Sugar, D. Toussaint, and R. S. VandeWater (MILC Collaboration), Phys. Rev. D 87, 054505 (2013).

[108] A. Hasenfratz and F. Knechtli, Phys. Rev. D 64, 034504 (2001).

[109] S. Mondal, R. Gupta, S. Park, B. Yoon, T. Bhattacharya, and H.-W. Lin, Phys. Rev. D 102, 054512 (2020).

[110] Y.-C. Jang, R. Gupta, H.-W. Lin, B. Yoon, and T. Bhattacharya, Phys. Rev. D 101, 014507 (2020).

[111] R. Gupta, B. Yoon, T. Bhattacharya, V. Cirigliano, Y.-C. Jang, and H.-W. Lin, Phys. Rev. D 98, 091501(R) (2018).

[112] H.-W. Lin, R. Gupta, B. Yoon, Y.-C. Jang, and T. Bhattacharya, Phys. Rev. D 98, 094512 (2018).

[113] R. Gupta, Y.-C. Jang, B. Yoon, H.-W. Lin, V. Cirigliano, and T. Bhattacharya, Phys. Rev. D 98, 034503 (2018).

[114] R. Gupta, Y.-C. Jang, H.-W. Lin, B. Yoon, and T. Bhattacharya, Phys. Rev. D 96, 114503 (2017).

[115] B. Yoon, Y. C. Jang, R. Gupta, T. Bhattacharya, J. Green, B. Joó, H. W. Lin, K. Orginos, D. Richards, S. Syritsyn, and F. Winter (Nucleon Matrix Elements (NME) Collaboration), Phys. Rev. D 95, 074508 (2017).

[116] T. Bhattacharya, V. Cirigliano, S. D. Cohen, R. Gupta, H.-W. Lin, and B. Yoon (Precision Neutron Decay Matrix Elements (PNDME) Collaboration), Phys. Rev. D 94, 054508 (2016).

[117] T. Bhattacharya, V. Cirigliano, R. Gupta, H.-W. Lin, and B. Yoon, Phys. Rev. Lett. 115, 212002 (2015).

[118] T. Bhattacharya, S. D. Cohen, R. Gupta, A. Joseph, H.-W. Lin, and B. Yoon, Phys. Rev. D 89, 094502 (2014).

[119] R. A. Briceno, H.-W. Lin, and D. R. Bolton, Phys. Rev. D 86, 094504 (2012).

[120] T. Bhattacharya, V. Cirigliano, S. D. Cohen, A. Filipuzzi, M. Gonzalez-Alonso, M. L. Graesser, R. Gupta, and H.-W. Lin, Phys. Rev. D 85, 054512 (2012).

[121] H.-W. Lin, Int. J. Mod. Phys. A 35, 2030006 (2020).

[122] G. S. Bali, B. Lang, B. U. Musch, and A. Schäfer (RQCD Collaboration), Phys. Rev. D 93, 094515 (2016).

[123] See Supplemental Material at http://link.aps.org/ supplemental/10.1103/PhysRevLett.127.182001 for more details on the methodology, technical aspects of the calculation and figures showing intermediate results, which includres Refs. [124,125].

[124] A. V. Radyushkin, Phys. Rev. D 96, 034025 (2017).

[125] M. Luscher and U. Wolff, Nucl. Phys. B339, 222 (1990).

[126] B. Yoon, R. Gupta, T. Bhattacharya, M. Engelhardt, J. Green, B. Joó, H.-W. Lin, J. Negele, K. Orginos, A. Pochinsky, D. Richards, S. Syritsyn, and F. Winter (Nucleon Matrix Elements (NME) Collaboration), Phys. Rev. D 93, 114506 (2016).

[127] X. Ji, Y. Liu, A. Schäfer, W. Wang, Y.-B. Yang, J.-H. Zhang, and Y. Zhao, Nucl. Phys. B964, 115311 (2021). 
[128] X.-D. Ji, J. Phys. G 24, 1181 (1998).

[129] P. Hagler, Phys. Rep. 490, 49 (2010).

[130] C. Alexandrou, S. Bacchio, M. Constantinou, P. Dimopoulos, J. Finkenrath, R. Frezzotti, K. Hadjiyiannakou, K. Jansen, B. Kostrzewa, G. Koutsou, C. Lauer, and C. Urbach (Extended Twisted Mass Collaboration), Phys. Rev. D 101, 034519 (2020).

[131] G. S. Bali, S. Collins, M. Göckeler, R. Rödl, A. Schäfer, and A. Sternbeck, Phys. Rev. D 100, 014507 (2019).

[132] C. Alexandrou, M. Constantinou, K. Hadjiyiannakou, K. Jansen, C. Kallidonis, G. Koutsou, and A. Vaquero AvilesCasco, Phys. Rev. D 96, 034503 (2017).

[133] J. R. Green, J. W. Negele, A. V. Pochinsky, S. N. Syritsyn, M. Engelhardt, and S. Krieg, Phys. Rev. D 90, 074507 (2014).

[134] N. Hasan, J. Green, S. Meinel, M. Engelhardt, S. Krieg, J. Negele, A. Pochinsky, and S. Syritsyn, Phys. Rev. D 97, 034504 (2018).
[135] E. Shintani, K.-I. Ishikawa, Y. Kuramashi, S. Sasaki, and T. Yamazaki, Phys. Rev. D 99, 014510 (2019).

[136] C. Alexandrou, S. Bacchio, M. Constantinou, J. Finkenrath, K. Hadjiyiannakou, K. Jansen, G. Koutsou, and A. V. Aviles-Casco, Phys. Rev. D 100, 014509 (2019).

[137] Y.-C. Jang, T. Bhattacharya, R. Gupta, H.-W. Lin, and B. Yoon (PNDME Collaboration), Proc. Sci., LATTICE2018 (2018) 123.

[138] T.-J. Hou et al., Phys. Rev. D 103, 014013 (2021).

[139] M. Burkardt, Int. J. Mod. Phys. A 18, 173 (2003).

[140] C. Alexandrou, K. Cichy, M. Constantinou, J. R. Green, K. Hadjiyiannakou, K. Jansen, F. Manigrasso, A. Scapellato, and F. Steffens, Phys. Rev. D 103, 094512 (2021).

[141] R. G. Edwards and B. Joo (SciDAC, LHPC, and UKQCD Collaborations), Nucl. Phys. B Proc. Suppl. 140, 832 (2005). 